\title{
Radioembolization of hepatic malignancies: evolving practice guidelines, techniques, and applications
}

\begin{abstract}
Purpose: Radioembolization (RE) is a type of intra-arterial locoregional therapy utilizing resin or glass microspheres in the management of primary and secondary hepatic malignancies. Using out instructional experience, the relative clinical efficacy of RE for the treatment of liver metastases is compared and contrasted to that of hepatocellular carcinoma (HCC). In addition, a literature review is presented to illustrate technique, clinical applications, and current research in liver directed radiation therapy.
\end{abstract}

Materials and Methods: A systematic review of the literature was performed emphasizing the clinical efficacy of RE for patients with HCC relative to metastatic liver disease. In addition, a 15 year institutional experience is presented. The institutional review board approved this retrospective analysis. Comparisons and contrasts are drawn between the existing literature data and our institutional data.

Results: Radioembolization is an important locoregional intra-arterial procedure utilizing microspheres for the management of hepatocellular carcinoma. Important technical considerations include: microsphere selection, identification and management of variant vascular anatomy, and periprocedural dosimetry. Two types of microspheres used: resin and glass microspheres. SIR-Spheres are a type of resin microsphere currently used at our institution. Radioembolization has been shown to prolong survival for patients with inoperable HCC. In addition, radioembolization is shown to be effective in the treatment of hepatic metastatic disease such as colorectal and neuroendocrine. Regarding the application of radioembolization to hepatic metastat.

Conclusion: Radioembolization is important for the management of HCC and metastatic liver disease. Due to the heterogeneity in treatment response between patients and disease, more research is necessary to uncover the molecular etiology governing treatment response.
Volume 7 Issue 6 - 2017

\author{
Kingsbury JM, Clark MR, Grammer RW, \\ Steadman BT, McCluskey KM, Higgins LH \\ West Virginia University, USA
}

Correspondence: Kingsbury JM, West Virginia University, I Medical Center Drive Morgantown,WV 26505, Tel 304-28265।5, Email jkingsbury@hsc.wvu.edu

Received: February 22, 2017 | Published: April 06, 2017

\section{Introduction}

Locoregional therapies are critical for managing hepatic malignancies of primary and metastatic origins. Currently the two main methods available to interventional radiologists for treatment of liver tumors are percutaneous ablation and intra-arterial therapies. Liver-directed intra-arterial therapies are considered palliative treatment options for patients with hepatic malignancies that are unresectable or resistant to chemotherapy. These intra-arterial therapies include radioembolization, transarterial bland embolization, transarterial chemoembolization (TACE) and drug-eluting bead TACE. These therapies are intended to occlude blood vessels feeding the tumor (TAE), directly deliver chemotherapy (TACE), or transport a radioactive isotope (radioembolization) to the targeted tumor to induce ischemia and eventual necrosis. ${ }^{1}$ Our aim is to perform an in-depth literature using medical electronic databases to review the most recent and relevant articles regarding the current techniques and technologies used in radioembolization and how it may continue to evolve.

\section{Technical considerations}

Radioembolization is performed via transcatheter intra-arterial administration of a radioactive isotope such as yttrium-90, which is incorporated into millions of microparticles. Once these microparticles are injected into the hepatic artery, they distribute according to particlefluid dynamics of the local vascularity and provincially lodge about the neoplasm. Ideally, the largest dose of radiation will be delivered to the targeted tumor with relative sparing of surrounding liver and other normal off-target tissues.

There are two types of microparticles or microspheres currently available: resin microspheres (SIR-Spheres) and glass microspheres. Each has unique characteristics which affect their intra-vascular distribution, therapeutic index, and complication rates. Resin microspheres measure 25-60 micrometers in diameter and are impregnated with the radioisotope while glass microspheres measure 20-30 micrometers with the radioisotope incorporated into the glass. In a $3-\mathrm{GBq}$ vial of microspheres, there are 40 million to 80 million resin microspheres compared to 1.2 million glass microspheres. ${ }^{2-4}$ Therefore, resin microspheres are more vulnerable to risks of stasis and reflux into the gastroduodenal artery (GDA) which can lead to off target radiation-induced complications.

The liver receives blood supply from both the hepatic artery and portal vein. Hepatic malignancies such as hepatocellular carcinoma (HCC) are predominantly supplied by the hepatic artery. ${ }^{5}$ Therefore, selective catheter placement in the hepatic arterial system is employed for radioembolization and the other intra-arterial therapies. It is thus important to have preemptive knowledge of each patient's vascular anatomy and to be able to recognize anatomic variants. Some variants include congenital accessory hepatic arteries, replaced hepatic arteries, and parasitized non-hepatic arteries recruited by hepatic tumors. Other variant anatomy that may be encountered is the blood supply of segment IV provided by the middle hepatic artery or origination of the middle hepatic artery from the left, right, or proper hepatic arteries. ${ }^{5}$ 
Additionally, the patient's specific arterial supply to each respective lobe of the liver should be elucidated. ${ }^{3-5}$ This can be performed through catheter-directed CT with selective contrast injection which allows determination of the volume of flow at the target area intended for treatment. ${ }^{5}$ This also allows for delineation of variant anatomy or any iatrogenic or pathologic distortions that may have previously occurred. Application of techniques to redirect blood flow can maximize microsphere distribution to the tumor while minimizing off target deposition (Figure 1-Figure 4).

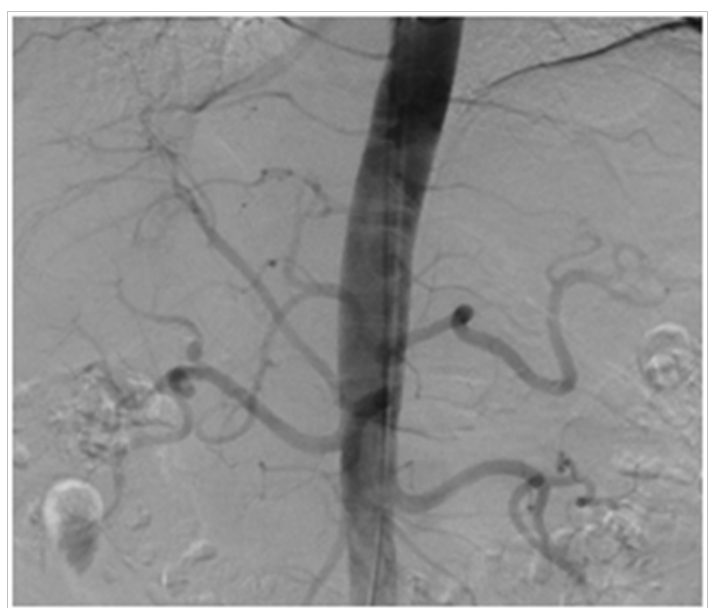

Figure I Aortogram demonstrating a replaced right hepatic artery.

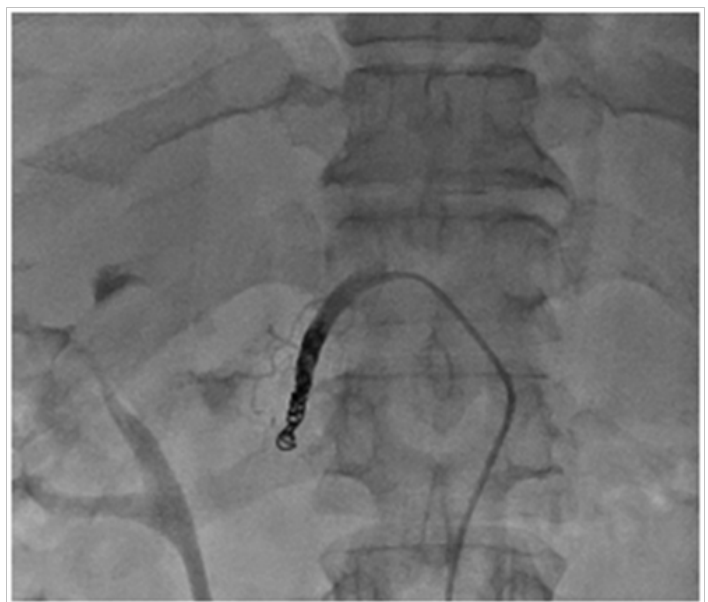

Figure 2 Coiling of the gastroduodenal artery (GDA).

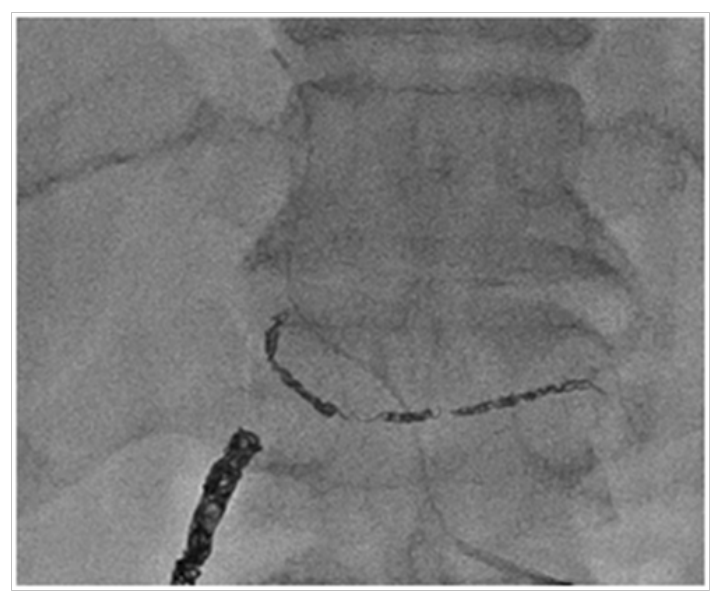

Figure 3 Additional coiling of the left gastric artery.

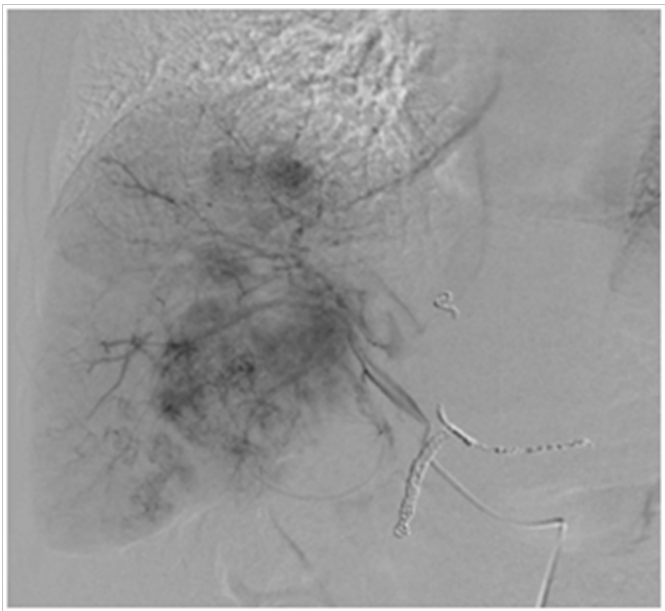

Figure 4 Subsequent angiogram demonstrates vascular redistribution and tumor burden.

\section{Procedure}

Planning angiography is critical to map the vascular anatomy and to identify and demarcate blood supply between the liver and target tumor. At this time preemptive embolization for prevention of extrahepatic deposition and quantification of hepatopulmonary shunt fraction can also be performed.

Determination of theoretical off target blood flow and thus microparticle deposition, or shunt fraction, is performed through the use technetium-99m macroaggregated albumin (MAA). The calculation of the hepatopulmonary shunt is important to exclude extrahepatic deposition which could result in off target organ injury. ${ }^{2-4}$ Further utility of pre-treatment MAA is discussed later on in this article.

Depending on the volumetric burden and distribution of the liver cancer and each patient's clinical status, targeting strategies may vary. If the patient is generally healthy with relatively robust liver intact they can tolerate larger treatment volumes of the liver or sequential lobar injections, as in the case of diffuse metastatic disease. Otherwise, precise targeting of individual segments allows the dose to be concentrated within an isolated tumor burden while sparing healthy liver parenchyma in the unaffected segments. ${ }^{2,4}$

At many institutions and throughout the literature, it is routine practice to prophylactically embolize the GDA and/or right gastric artery to prevent reflux of the radioactive microparticles and subsequent gastrointestinal injury. However, prophylactic embolization presents with its own complications such as subsequent formation of difficult to embolize collaterals, coil migration, recanalization, and arterial dissection. ${ }^{2,3,5}$

Due to these potential complications, there has been a decline in the prophylactic embolization of the GDA as glass microspheres have been safely used for radioembolization as long as certain premonitory steps are taken: skeletonization of distal hepaticoenteric anastomoses, flushing with dextrose solution, use of precalibration high-activity microspheres, and use of ARMC. ${ }^{3}$

Tumor response is assessed with multi-phase contrast-enhanced computed tomography (CT) or magnetic resonance imaging (MRI). Whichever modality is chosen, it should be consistent throughout the course of care to ensure accuracy. Imaging follow-up is 
recommended at 1-3 months and at 3-6 month intervals thereafter. ${ }^{1}$ Oftentimes follow-up imaging at 1 month is performed but this is often considered premature as it commonly precedes any imaging manifestation of radioembolization responsiveness. ${ }^{1,2}$ A favorable radiologic evaluation of the tumor's response to therapy has been shown to correlate with survival outcomes. ${ }^{1}$ Changes or decreases in tumor size are a generally accepted radiological marker for assessing treatment response but can be of limited utility in some circumstances. Response evaluation criteria for solid tumors include the World Health Organization (WHO), RECIST (Response Evaluation Criteria in Solid Tumors), European Association for the Study of the Liver (EASL), and modified RECIST (MRECIST) to name a few. These are methods to assess treatment response including imaged based measurements and additional methodology that incorporates necrosis, PET scans, and tumor density. ${ }^{1}$

However, as mentioned these traditional anatomic size criteria are limited for such evaluations as they can be confounded by tumor necrosis. Additionally, as mentioned above, anatomic changes such as a decrease in size lag behind treatment induced functional and metabolic changes that would otherwise indicate a positive response. Furthermore, treatment induced tumor ischemia and hypoxia with resultant necrosis and hemorrhage can present as a paradoxical increase in tumor size in the early stages of follow-up imaging. Functional imaging modalities such as diffusion weighted (DW) MRI and positron emission tomography (PET) imaging, can be used to assess tumor response in patients with primary and metastatic hepatic malignancies. ${ }^{1}$ These functional imaging techniques offer the advantage of earlier signals of treatment response at the cellular/ metabolic level, prior to any obvious anatomic changes.

Currently, quantitative DW (diffusion weighted) MRI and PET/ CT measurements are being developed to monitor early treatment response. ${ }^{1} \mathrm{DW}$ imaging measures the random movement of water molecules within a pre-calculated volume of tissue, which is then used to quantify the apparent diffuse coefficient (ADC) to give the magnitude of this movement. ${ }^{1}$ Past studies have shown that ADC values can helpful for distinguishing viable from necrotic tissues. HCC and liver metastases typically have low ADC values and necrotic tissues have elevated ADC values, distinguishing viable tumor from necrosis. ${ }^{1}$ These increases in ADC values can be seen much earlier compared to changes in anatomic size of lesions. However these prior studies lack reproducibility and variability. Therefore, a standardized DW MRI protocol with larger sample size is warranted. ${ }^{1}$

Fluorodeoxyglucose (FDG) positron emission tomography is an imaging technique that assesses tumor response to radioembolization through measuring its metabolic activity, but is generally not useful with HCC because HCC does not take up FDG adequately. ${ }^{1}$ Similar to DW imaging, studies have found that PET or PET/CT can detect more and earlier responses to therapy than anatomic or necrosis criteria. ${ }^{1}$

\section{Dosimetry}

To individualize treatment planning and optimize outcomes, it is prudent to attempt to predict the absorbed dose received by the tumor and the absorbed dose that may be distributed to healthy liver and lungs. ${ }^{4}$ Such image-based dosimetry may be performed before, during, or after treatment. Before every radioembolization procedure a scout dose of technetium-99m macroaggregated albumin (99mTcMAA) is administered to simulate the expected biodistribution of the main therapy, Y-90. Single photon emission computed tomography (SPECT) combined with CT is the preferred modality to elucidate the biodistribution of 99m-Tc-MAA within and around the liver. ${ }^{2-4}$ In addition, this allows for the calculation of the shunted blood to the lungs so as to avoid off target deposition and subsequent radiation Pneumonitis. ${ }^{3,4}$ In the case of significant shunting to the lungs, the prescribed activity is empirically reduced in the following manner: $10-15 \%$ shunt fraction; $20 \%$ activity reduction; $15-20 \%$ shunt fraction: $40 \%$ activity reduction; $>20 \%$ shunt fraction: no treatment. ${ }^{4}$ However, the accuracy of $99 \mathrm{mTc}-\mathrm{MAA}$ is questionable as the lung shunt fraction may be overestimated due to the presence of free pertechnetate. Also, the variability in MAA particle size may affect the accuracy and reproducibility of the lung shunt fraction. However if the $99 \mathrm{mTc}-\mathrm{MAA}$ scout dose confirms a patient's eligibility for radioembolization, the scout dose may also be used for treatment planning.

Activity planning or dose calculation for the target tumor or liver tissue volume can be performed using the body surface area method (BSA), the Medical Internal Radiation Dose (MIRD) formula, or anatomic partition models. ${ }^{2,4}$ The BSA method generally correlates with liver volume and the administered activity can be adjusted. This is currently the preferred method for calculating prescribed activity in patients with multiple liver metastases to be treated with resin microspheres. ${ }^{2,4}$ The standard BSA formula for resin microspheres is based on the BSA and the fraction of the total liver volume involved by the tumor. ${ }^{2}$ A weakness of the BSA method is that it if there is a disproportional tumor-to-liver ratio or added volume of the tumors then accuracy suffers and highly variable absorbed doses between patients may be encountered. For example, large patients with small livers will receive relatively high doses and vice versa. ${ }^{2,4}$

The MIRD formula is used in the dose calculation for glass microspheres and is based on the estimation of the mean absorbed dose in the target liver volume. ${ }^{2}$ According to the MIRD method for glass microspheres, the treating radiologist prescribes an amount of activity based on a desired liver absorbed dose which is largely dependent upon the patient's clinical status. However, the intrahepatic biodistribution varies and the dose received by the tumor and healthy liver also varies between patients. This method can be useful for radiation with a segmental distribution in patients with disease limited to $<2$ liver segments. ${ }^{2}$ These patients are treated with supratherapeutic doses to a target volume that is inclusive of both the tumor and the normal liver parenchyma, which will suffer ablation while sparing the untreated part of the liver. ${ }^{2}$ When using resin microspheres for radioembolization, dose calculation is based on a whole-liver infusion. Therefore, the dose is represented as a target site percentage of the whole liver activity.

Neither of the aforementioned dose calculation methods account for a heterogeneous distribution of microspheres and radiation to both tumors and provincial liver parenchyma. An answer to this challenge is the 'partition' method, which involves preselecting absorbed doses with a maximum activity that is within safe limits, based on preemptive anatomic imaging and anticipated radioembolic activity. ${ }^{2,4}$ However, this method can be prone to error and cumbersome because of inter-user variability. ${ }^{2,4}$

Patients traditionally relegated to radioembolization present with a diffuse and infiltrative hepatic tumor burden which is problematic for segmentation and preplanning given the $99 \mathrm{mTc}-$ MAA distribution. ${ }^{1,2,4}$ A considered resolution is a dual tracer SPECT technique combining 99mTc-MAA SPECT for simulation of Y90 activity distribution and $99 \mathrm{mTc}$-sulfur colloid SPECT for evaluation of functional liver parenchyma. ${ }^{1}$ The sulfur colloid is used to delineate normal liver 
tissue and its distribution thusly revealed with a second SPECT. The absorbed dose to normal liver parenchyma and the tumor can then be calculated, therefore. The end result is physiology based functional imaging for hepatic radioembolization treatment planning and optimized patient care.

\section{Future directions: the road to personalized radioembolization therapy}

In the near future, microparticles could be engineered to target malignant cells based on molecular attributes such tumor cell surface markers. During planning angiography, these newly tailored microparticles could be delivered simultaneously and radiolabeled with gamma emitting radiotracers such as $67 \mathrm{Co}, 111 \mathrm{In}$, and 123I via the right and left hepatic arteries. Subsequent SPECT-CT imaging would not only allow for pulmonary and hepatic shunt fraction measurements, but also confirm and highlight tumor selective uptake of the microparticles. A manifold of cell surface markers with attached gamma emitters would need to be administered in a trial fashion to elucidate the gamma emitter with the most potent yet accurate dose estimates. This would allow for selection of the most appropriate therapy while maximizing the targeted tumor to liver dose ratio for each patient and their respective cancer. Furthermore, refinement of the fluid-particle dynamics of the radioactive microparticles would also be greatly beneficial. An ideal particle for planning angiography and dosimetry would follow a distribution pattern that is very similar to the distribution pattern of the actual microspheres used for treatment, a mutuality not endorsed between 99Tc-MAA and Y90 microspheres. The end result being integration of multiple steps consolidated into a single session for the patient. ${ }^{6}$

Similar to this strategy, other microparticles could also be engineered to inhibit cellular mediation, growth signaling, and other gene regulated pathways. This could serve as a premonitory insult to the tumor cell, a form of radiosensitization which would amplify the impact of subsequent radioembolic therapies. The only foreseen limitation would be a saturation of cell surface markers and therefore potentially loose, unbound particles. Efforts have been made utilizing the aforementioned principles, notably the transfer of plasmid DNA resulting in immunomodulation to sensitize radioresistant melanoma tumors. ${ }^{7}$ This concept could be applied to radioembolization. Furthermore, research is currently underway exploring the utility of gold nanoparticles for the targeted delivery of gene therapy to tumors. ${ }^{8}$ With the above methods in place, prophylactic embolization of the GDA and other hepatic vessels would no longer be necessary. With such precise partitioning of the radioactivity based on catheter selection and cell surface attraction, the unwanted off-target side effects of contemporary radioembolization such as radiation-induced gastrointestinal embolization would be a thing of the past.

To build upon the idea of presensitization, a conjoined effort of TACE and radioembolization would allow for a more robust assault on cancer, thwarting further growth while shattering its DNA, forcefully persuading the tumor to suffocate in its own cellular debris. To our knowledge this methodology has not been applied dually against isolated HCC. Weakly homologous attempts have been made against biliary tract cancer ${ }^{9}$ and metastatic colorectal cancer ${ }^{10}$ which shows promise but a need for further work. A practical approach may be to suspend cancerous cells in a broth of TACE agents and to observe their course with and without additive impregnation of radiospheres. The challenge of this in vitro approach, of course, is to accurately quantify and qualify in meaningful terms their smoldering demise.

\section{Conclusion}

Continued research and future developments in radioembolization will continue to refine its technique, technology, and utility and as an important component in management may someday lead the therapy of hepatic malignancies. We must further uncover the molecular etiology governing the robustness of the malignancy in mind, from patient to patient and cancer to cancer in an effort to provide truly personalized care, personalized to the level of the gene.

\section{Funding}

None.

\section{Acknowledgements}

None.

\section{Conflicts of interests}

The authors declare that there is no conflict of interest.

\section{References}

1. Guo Y, Yaghmai V, Salem R, et al. Imaging tumor response following liver-directed intra-arterial therapy. Abdom Imaging. 2013;38:12861299.

2. Padia SA, Lewandowski RJ, Johnson GE, et al. Radioembolization of Hepatic Malignancies: Background, Quality Improvement Guidelines, and Future Directions. J Vasc Interv Radiol. 2017;28(1):1-15.

3. Ward T, Louie J, Sze DYttriu. m-90 Radioembolization with Resin Microspheres without Routine Embolization of the Gastroduodenal Artery. J Vasc Interv Radiol. 2017;28(2):246-253.

4. Smits ML, Elschot M, Sze DY, et al. Radioembolization Dosimetry: The Road Ahead. Cardiovasc Intervent Radiol. 2015;38(2): 261-269.

5. Shah R, Sze D. Radioembolization: Identifying and Managing Anatomic Variants. Liver Radioembolization with Y90 Microspheres, Medical Radiology. Diagnostic Imaging. 2013:886.

6. Lam MG, Goris ML, Iagaru AH, et al. Prognostic Utility of Y90 Radioembolization Dosimetry Based on Fusion $99 \mathrm{mTc}-$ Macroaggregated Albumin-99mTc-Sulfur Colloid SPECT. J Nucl Med. 2013;54(12):2055-2061

7. Savarin M, Kamensek U, Cemazar M, et al. Electrotransfer of plasmid DNA radiosensitizes B16F10 tumors through activation of immune response. Radiol Oncol. 2017;51(1): 30-39.

8. Mendes R, Fernandes AR, Baptista PV. Gold Nanoparticle Approach to the Selective Delivery of Gene Silencing in Cancer-The Case for Combined Delivery?. Genes. 2017;8(3):pii. E94.

9. Marisco V, Vasile E, Brandi G, et al. Transarterial Chemo-Embolization (TACE) and Radio-Embolization (TARE) in the combined modality treatment of advanced biliary tract cancer (aBTC): evaluation of feasibility and activity. Ann Oncol. 2015;26:iv32-iv33.

10. DeSouza A, Daly K, Yoo J, et al. Safety and efficacy of combined yttrium 90 resin radioembolization with Aflibercept and FOLFIRI in a patient with metastatic colorectal cancer. Case Reports in Oncological Medicine. 2015(2015):6. 